MICROLOCALIZACIÓN DE Phytelephas aequatorialis Spruce EN LOS PREDIOS DE LA GRANJA EXPERIMENTAL ANDÍL

\title{
MICROLOCALIZACIÓN DE Phytelephas aequatorialis Spruce EN LOS PREDIOS DE LA GRANJA EXPERIMENTAL ANDÍl, ORIENTADA A SU COMERCIALIZACIÓN
}

\author{
AUTORES: John Ricardo Pin Cedeño ${ }^{1}$ \\ Alfredo Jimenez González ${ }^{2}$ \\ Marcos Pedro Ramos Rodríguez ${ }^{3}$ \\ Otto Francisco Mero Jalca4 \\ Cesar Alberto Cabrera Verdesoto 5
}

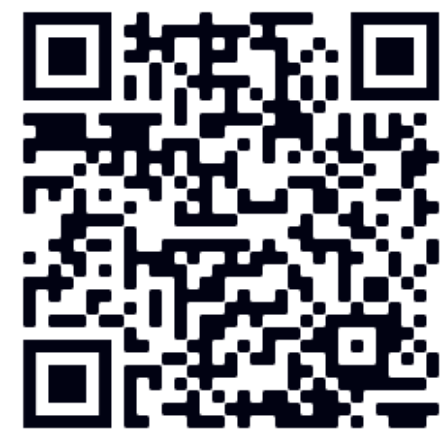

DIRECCIÓN PARA CORRESPONDENCIA:alfredo.jimenez@unesum.edu.ec

Fecha de recepción: 12/06/2019

Fecha de aceptación: 07/11/2019

RESUMEN

Debido a vacíos en el conocimiento relacionado con inventarios y microlocalización de Phytelephas aequatorialis en Ecuador, se desconoce la cantidad de especies y/o individuos existentes, y ante la alta demanda del producto se hace prioritario inventariar la especie de manera global, para conocer sobre las potencialidades y usos sustentables de esta planta. Por lo antes mencionado, se realizó un estudio sobre los usos y potencialidades de este taxa, en los predios de la Granja Experimental de Andil. Mediante un muestreo aleatorio simple, se inventariaron 30 palmas al azar de la especie objeto de estudio, para lo cual se midieron, la morfología del tallo, apariencia general/forma de la copa y la inflorescencia. Así se tuvo en cuenta variables ambientales relacionadas con la intervención humana. Por medio de encuestas realizadas a tres grupos; agricultores, centros de acopio y talleres de

\footnotetext{
${ }^{1}$ Ingeniero Forestal. Empresa Pública. Universidad Estatal del Sur de Manabí. Km 1 1² Vía Novoa s/n Campus Los Ángeles, Jipijapa, Manabí, Ecuador. CP. 130 650, e-mail: rickys_29@hotmail.com

Dr. En Ciencias Forestales, Carrera de Ingeniería Forestal, Universidad

${ }^{2}$ Dr. En Ciencias Forestales, Carrera de Ingeniería Forestal, Universidad Estatal del Sur de Manabí. Km 1 1² Vía Novoa s/n Campus Los Ángeles, Jipijapa, Manabí, Ecuador. CP. 130 650, e-mail: alfredo.jimenez@unesum.edu.ec, teléf.: http//orcid.or/0000-0002-1768-5566. Telf. 593+996163589.

${ }^{3}$ Dr. En Ciencias Forestales, Carrera de Ingeniería Forestal, Universidad Estatal del Sur de Manabí. Km 1 1 1⁄2 Vía Novoa s/n Campus Los Ángeles, Jipijapa, Manabí, Ecuador. CP. 130 650, e-mail: marcos.ramos@unesum.edu.ec,

${ }^{4}$ Ingeniero Forestal. Carrera de Ingeniería Forestal, Universidad Estatal del Sur de Manabí. Km 1 1⁄2 Vía Novoa s/n Campus Los Ángeles, Jipijapa, Manabí, Ecuador. CP. 130 650, e-mail: otto.mero@unesum.edu.ec

${ }^{5}$ Ingeniero Forestal. Carrera de Ingeniería Forestal, Universidad Estatal del Sur de Manabí. Km 1 1⁄2 Vía Novoa s/n Campus Los Ángeles, Jipijapa, Manabí, Ecuador. CP. 130 650, e-mail: cesar.cabrera@unesum.edu.ec
} 
John Ricardo Pin Cedeño, Alfredo Jimenez González, Marcos Pedro Ramos Rodríguez...

manufactura y comercialización, se pudo determinar entre otras cosas, que los recursos de la tagua en el sector no son correctamente manejados, en los centros de acopios, la llegada de materia prima es intermitente, en tanto que en Sosote de Rocafuerte la demanda de materia prima de los comerciantes de artesanías es alta. La intervención de las autoridades y la academia juegan un papel indispensable para poder impulsar del desarrollo de las actividades que van desde talleres comunitarios, asociaciones, hasta capacitaciones del artesano que permitan desarrollar las potencialidades y el correcto manejo de la tagua en el sector desde la plantación hasta la manufactura.

PALABRAS CLAVE: bosques, especies, usos, aprovechamiento, comercialización.

\section{MICROLOCALIZATION OF Phytelephas aequatorialis Spruce IN THE PREMISES OF THE ANDÍl EXPERIMENTAL FARM, ORIENTED TO ITS COMMERCIALIZATION}

\section{ABSTRACT}

Due to gaps in the knowledge related to inventories and microlocation of Phytelephas aequatorialis in Ecuador, the amount of existing species and / or individuals is unknown, and in view of the high demand of the product it is a priority to inventory the species in a global manner, to know about the potential and sustainable uses of this plant. For the aforementioned, a study was carried out on the uses and potentialities of this taxa, in the lands of the Experimental Farm of Andil. By using a simple random sampling, 30 palms were randomly inventoried from the species under study, for which the stem morphology, general appearance / shape of the crown and the inflorescence were measured. Thus, environmental variables related to human intervention were taken into account. Through surveys made to three groups; Farmers, collection centers and workshops of manufacture and commercialization, it was possible to determine among other things that in the sector the resources of the tagua are not properly handled, in the collection centers, the arrival of raw material is intermittent, while at Sosote in Rocafuerte the raw material demand of the craft merchants is high. The intervention of the authorities and the academy play an indispensable role in order to promote the development of activities ranging from community workshops, associations, to training of the craftsman to develop the potential and proper management of the tagua in the sector from the plantation until the manufacturing.

KEYWORDS: forests, species, uses, exploitation, commercialization.

INTRODUCCIÓN

Los ecosistemas forestales albergan la mayor riqueza biológica de nuestro planeta y en ellos habitan más de 1200 millones de personas que dependen de los bosques y selvas como su principal medio de vida. Irónicamente más del $90 \%$ de estas poblaciones sufren de niveles elevados de pobreza (Marshall, Schreckenberg \& Newton, 2006). 

EXPERIMENTAL ANDÍL

De acuerdo con la agenda nacional de investigación sobre la biodiversidad, se reconoce a Ecuador como uno de los 20 Países Megadiversos afines. Precisamente, frente a la necesidad de encontrar alternativas al modelo de desarrollo basado en el extractivismo, la biodiversidad podría ser uno de los motores de esa nueva bioeconomía, al ofrecer oportunidades para la producción, transformación y comercialización de productos derivados de la biodiversidad que son demandados por el sector industrial, comercio y servicios. Para ello, es fundamental que las instituciones del sector público, privado, académico, empresarial y financiero acuerden la ruta a seguir en los próximos años en materia de investigación, innovación y desarrollo tecnológico (INABIO, 2017).

Por otra parte, las diversas actividades de las comunidades en relación con la explotación y manejo de bosques y recursos forestales, es cada vez más relevante. Actualmente se promueve en el mundo "como una de las estrategias para lograr el manejo forestal sostenible, equitativo y participativo". (Eke et al., 2016, p. 25)

Debido a vacíos en el conocimiento relacionado con inventarios y microlocalización de Phytelephas aequatorialis en Ecuador, se desconoce la cantidad de especies y/o individuos existentes, y ante la alta demanda del producto se hace prioritario inventariar la especie de manera global, para conocer sobre las potencialidades y usos sustentables de esta planta. Así el objetivo del trabajo consiste en: microlocalizar especímenes de Phytelephas aequatorialis Spruce en los predios de la granja Experimental Andil, orientada a su comercialización.

Para la obtención de datos relacionados con la recolección, los centros de acopio, el procesamiento y la comercialización de los productos que brinda esta palma, se aplicaron tres tipos de encuestas, la primera dirigida a indagar sobre sobre el conocimiento que tienen los habitantes de Andil sobre aspectos etnobotánicos de la especie objeto de estudio; la segunda encuesta estuvo dirigida a las personas involucradas con el procesamiento de las semillas de la planta objeto de estudio en sectores de Manabí; y la tercera encuesta estuvo direccionada a la comercialización de productos, artesanía y otros derivados de la tagua.

\section{DESARROLLO}

\section{Materiales y métodos}

Ubicación geográfica del inventario

La investigación se realizó en la comunidad Andil, la cual se encuentra ubicada a 4,60 km desde el centro de la ciudad de Jipijapa, por la avenida universitaria, puntualmente en el noreste de Jipijapa, cantón ubicado al sur de la provincia de Manabí

\section{Vegetación}

En la región predomina el Bosque deciduo de tierras bajas, y el bosque semideciduo montano bajo o pie montano, descritos por MAE (2012); Grijalva et al., (2012); Jimenez et al., (2017) y Valencia et al., (2013), han reportado a Phytelephas aequatorialis como un árbol del Bosque de litoral húmedo hasta bosque litoral piemontano: 0-1500 m, y su distribución geográfica lo ubican en las provincias de Cañar, Chimborazo, Cotopaxi, Esmeraldas, Guayas, Manabí y Pichincha.

\section{Metodología}


John Ricardo Pin Cedeño, Alfredo Jimenez González, Marcos Pedro Ramos Rodríguez...

Se utilizó el método empírico del conocimiento, porque "este tipo de conocimiento se caracteriza principalmente por un enfoque que se basa en la experiencia y que responde directamente a una $\mathrm{u}$ otra demanda social, a una u otra necesidad práctica" (Suárez, 2011, p 112).

A través de encuestas de acuerdo con los criterios de (Aguirre et al., 2014; Jimenez et al., 2017), con modificaciones del autor. En este trabajo se realizaron tres tipos de encuestas: la primera, consiste en una encuesta etnobiológica y fue tomada a personas que habitan dentro de la comunidad de Andil, ubicados en la parroquia Jipijapa; la segunda encuesta, estuvo dirigida a personas que laboran en centros de acopio de semillas de tagua, y una tercera encuesta, fue realizada a procesadores y comerciantes de semillas y artesanías de Phytelephas aequatorialis, respectivamente. En el caso de las encuestas 2 y 3, se aplicó el método Likert.

Tamaño de la muestra

La encuesta se aplicó con el fin de conocer el uso y aprovechamiento de Phytelephas aequatorialis. Teniendo en cuenta las características de esta población, se hicieron preguntas sencillas de SI y NO con un grupo de variables. Una vez que se conoció la población vinculada con las actividades extractivas (12 personas), se calculó el número de personas a encuestar en la comunidad, para lo cual se utilizó la fórmula planteada por Torres et al., (2006) según este autor, cuando se conoce el tamaño de la población, la muestra necesaria es más pequeña y su tamaño se determina mediante la fórmula [1]:

$n=\frac{N Z^{2} p q}{\left.(N-1) e^{2}+Z^{2} p q\right)}$

Dónde:

n: tamaño de la muestra que se necesita conocer.

$\mathrm{N}$ : tamaño conocido de la población

Z: valor de z, 1,96 para un nivel de confianza del $95 \%, \alpha=0,05$

pq: varianza de la población

Los cálculos determinaron que 10 encuestados representa el $83.33 \%$ de encuestas, datos aceptables para el neotrópico.

De acuerdo con Morales (2012), como la varianza de la población se desconoce, se coloca la varianza mayor posible porque a mayor varianza hará falta una muestra mayor. La varianza en los ítems dicotómicos (dos respuestas que se excluyen mutuamente) es igual a pq y la varianza mayor (la mayor diversidad de respuestas) se da cuando $\mathrm{p}=\mathrm{q}=0,50$ (la mitad de los sujetos responde sí y la otra mitad responde no) por lo que en esta fórmula [1] pq es siempre igual a $(0,50)(0,50)=$ 0,25 (es una constante). e: error que se prevé cometer. Y como no se requiere un error mayor del $3 \%$, se tiene que $\mathrm{e}=0,03$.

En el caso de las encuestas realizadas en los centros de procesamiento y en los comerciantes se le realizó al 90 \% de los trabajadores directos a la producción y a la venta, respectivamente.

Descripción de los instrumentos

La encuesta 1 estuvo dirigida a los moradores de la comunidad de Andil, y tuvo como objetivo, indagar sobre el conocimiento de Phytelephas aequatorialis en sectores de Manabí. La encuesta 2,

70 UNESUM-Ciencias. Publicación cuatrimestral. Vol. 3, Año 2019, No. 2 (Mayo - Agosto) 

EXPERIMENTAL ANDÍL

fue dirigida a procesadores de productos (semillas), y se realizó con el objetivo de indagar sobre el procesamiento de Phytelephas aequatorialis en sectores de Manabí. La tercera encuesta, se realizó a los comerciantes con el objetivo de indagar sobre la comercialización de Phytelephas aequatorialis en sectores de Manabí.

\section{Muestreo}

Para la microlocalización de palmas de Phytelephas aequatorialis, se realizaron recorridos de campo, así como conversatorios con líderes locales, campesinos y personal de la granja experimental, perteneciente a la Universidad Estatal del Sur de Manabí, además de entrevistar a los pobladores, se realizaron conversatorios con el encargado de la finca. Se realizó un muestreo aleatorio simple. Una muestra aleatoria o probabilística es aquella en la que todos los sujetos de la población han tenido la misma probabilidad de ser escogidos. Son en principio los tipos de muestra más profesionales (figura 1). El muestreo aleatorio simple es muy utilizado para poblaciones pequeñas, como es el caso de las 30 palmas que se muestrearon en Andil, según los criterios de Santos et al., (s/f), con modificaciones del autor, que consistieron en: realizar un muestreo de palmas al azar, en donde el colector escoge al azar 30 muestras de palmas y obtiene parámetros representativos por palma, en este caso se midieron los siguientes parámetros:

a) Morfología del tallo:

1. Medición del diámetro a $20 \mathrm{~cm}$ sobre el nivel del suelo $(\mathrm{cm})$

2. Medición del diámetro a 1,5 $\mathrm{m}$ de altura $(\mathrm{cm})$

3. Longitud (m) del tallo entre 11 cicatrices de hojas, medida comenzando desde la parte inferior de la cicatriz de la primera hoja hasta la parte inferior de la cicatriz de la undécima hoja.

b) Apariencia general/forma de la copa

\section{Esférica}

2. Semiesférica

3. Silueta en forma de " $X$ "

4. En forma de "V"

5. Otra (especificar)

c) Inflorescencia

1. Masculina

2. Femenina

En el caso de las variables ambientales que predominan en el área, se tuvo en cuenta dos categorías de intervención, a saber: Intervenidas y No Intervenidas. 


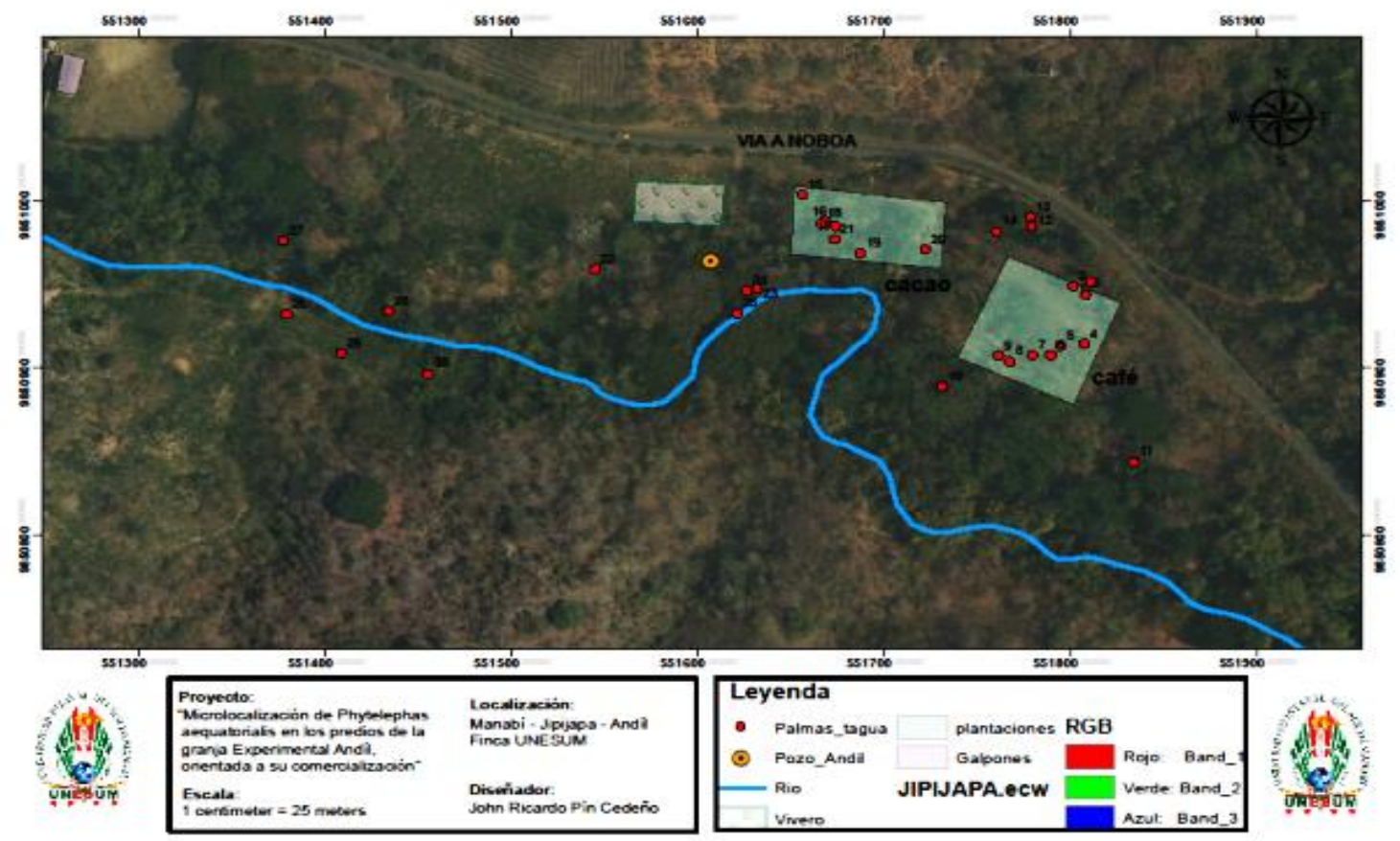

Figura 1. Ubicación de los sitios de muestreo en la comunidad Andil, parroquia Jipijapa, Manabí, Ecuador.

\section{Resultados y discusión}

Algunos autores han planteado que el desarrollo vegetativo de Phytelephas aequatorialis no ha sido investigado; sin embargo, observaciones indirectas sugieren que la tagua requiere 10 años para alcanzar el desarrollo morfológico completo (subadulto) y 14-15 años para llegar a la madurez sexual. Se calcula que un ejemplar de dos metros de alto no tiene menos de 35 a 40 años de edad. Los resultados de la variable altura en los especímenes inventariados en Andil, demuestran, que, los individuos no alcanzaron los $2 \mathrm{~m}$ de altura, no obstante, se comprobó que la altura total de las plantas alcanza hasta 5 m o más, lo que corrobora que estos taxa, poseen al menos 35 años de edad. La mayor cantidad de individuos se encontró con alturas ubicadas en la clase III.

En la figura 2 se observa la distribución de la altura de los individuos a 0,20 m y a $1.50 \mathrm{~m}$ de la altura del suelo donde podemos observar como los valores a distintas alturas forman líneas de rangos similares. 

EXPERIMENTAL ANDÍL

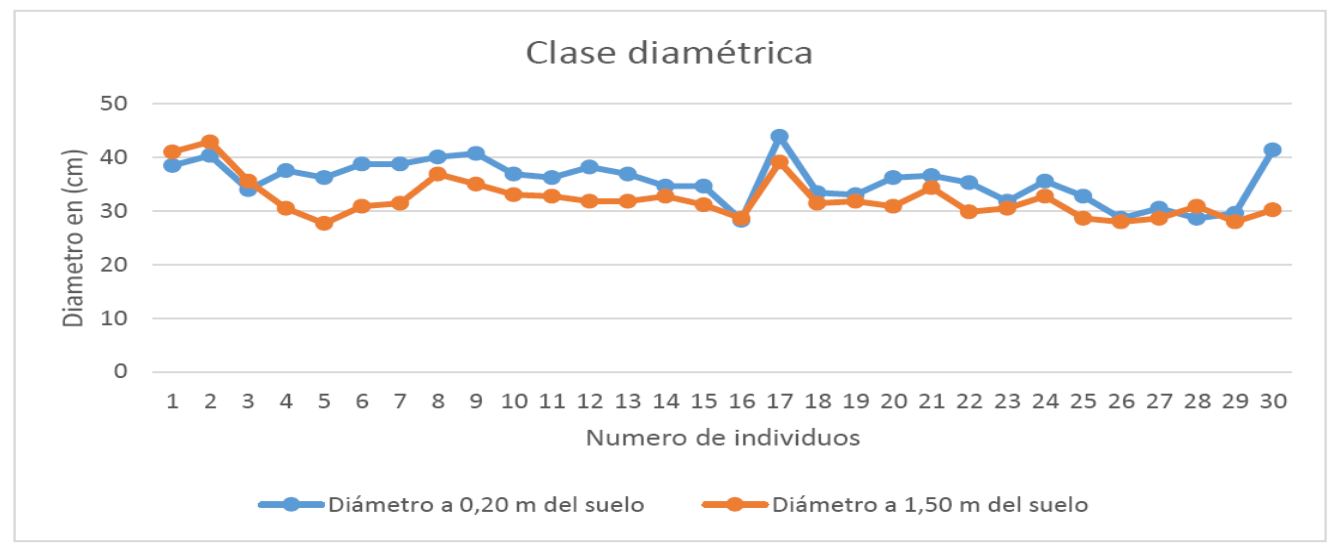

Figura 2. Representación gráfica de la altura de los individuos de Phytelephas aequatorialis, inventariados en Andil.

El diámetro es un indicador de utilidad, puesto que a través de éste se puede conocer la edad de los individuos de Phytelephas aequatorialis. Se ha confirmado que es una palmera dioica, solitaria con tallos café oscuros de 1-8 $\mathrm{m}$ de alto y $10-20 \mathrm{~cm}$ de diámetro, hojas erectas con folíolos en grupos de 4-7 y proyectados en varios planos de orientación (Montúfar et al., 2013). En la siguiente tabla se muestra la distribución del diámetro de los individuos inventariados en Andil (tabla 1).

Tabla 1. Distribución por clases diamétricas de los individuos de Phytelephas aequatorialis inventariados en los predios de Andil.

\begin{tabular}{|c|c|c|c|c|}
\hline $\begin{array}{l}\text { Clases } \\
\text { diamétricas }\end{array}$ & I & II & III & IV \\
\hline $\begin{array}{l}\text { Diámetro } \\
\text { (cm) }\end{array}$ & $\begin{array}{l}\text { cे } \\
\text { ì }\end{array}$ & $\begin{array}{l}n \\
\text { d } \\
\text { d }\end{array}$ & $\begin{array}{l}\text { P } \\
\text { ñ } \\
m\end{array}$ & $\begin{array}{l}\text { ? } \\
\text { f } \\
\text { f }\end{array}$ \\
\hline Abundancia & 4 & 8 & 13 & 5 \\
\hline
\end{tabular}

En relación con la estructura poblacional de la tagua, ésta varía en función del tipo de hábitat. En sistemas agroforestales, arboledas, pastizales o bosques secundarios, existe una alta densidad de juveniles (individuos $>1 \mathrm{~m}$ sin tronco visible) frente a adultos (individuos con tronco visible y con órganos reproductivos, hasta 30:1) pero hay una ausencia casi total de subadultos (individuos con troncos, pero sin órganos reproductivos), en el caso de las áreas muestreadas en Andil, los individuos de tagua se comportan de la siguiente manera:

Como la regeneración natural en la granja experimental Andil presentan comportamientos diferentes y esto se debe a los factores de intervención que las afectan, por lo consiguiente se dividió como sigue; por lugares donde hay intervención humana y lugares donde no hay intervención humana, al realizar el proceso de microlocalización y posterior recolección de datos para llenar el inventario se constató in situ que algunos individuos de Phytelephas aequatorialis se encuentran ubicados en lugares donde se desarrolló o se desarrollan aún actividades de intervención humana como plantaciones agrícolas, tala de bosque natural, pastizales y ganadería. 
John Ricardo Pin Cedeño, Alfredo Jimenez González, Marcos Pedro Ramos Rodríguez...

En estos sitios donde hubo o hay intervención humana es normal encontrarse con regeneración natural de la tagua, cabe recalcar que en estos lugares se observó que la regeneración natural alcanza una altura que oscila entre $10525 \mathrm{~cm}$ a $40 \mathrm{~cm}$, está claro que es producto del constante uso del suelo en las diferentes actividades humanas que se llevan a cabo en algunos sectores de la granja experimental Andil lo que impide que los nuevos individuos lleguen a alcanzar su etapa de madurez y esto pueda afectar a futuro la replicación de la especie. Las especies indicadoras de calidad ambiental se usan para evaluar los impactos que sufren los ecosistemas a causa de las actividades antropogénicas (Jimenez, 2012).

Combinar el uso de estas especies, junto con otras herramientas de evaluación podría mejorar la efectividad con la que se perciben y cuantifican los cambios en la biodiversidad debidos a las perturbaciones originadas por las actividades humanas (Isasi-Catalá, 2011).

En los lugares donde no hay intervención humana se pudo observar que la regeneración natural se lleva a cabo exitosamente y sin ningún tipo de inconveniente por lo que fueron encontrados individuos de Phytelephas aequatorialis en diferentes tamaños que van desde los $30 \mathrm{~cm}$ hasta los adultos que llegan a medir hasta unos $15 \mathrm{~m}$ a $20 \mathrm{~m}$ en su altura total.

A decir de Montúfar et al., 2013), han reportado densidades poblacionales de esta especie en dos sectores de la provincia de Manabí, a saber, Junín y Canuto. De acuerdo con estos autores la estructura poblacional de la tagua varía en función del tipo de hábitat. En sistemas agroforestales, arboledas, pastizales o bosques secundarios, como es el caso de Andil, donde, como refieren estos autores, hay una ausencia casi total de subadultos (individuos con troncos, pero sin órganos reproductivos). En contraste, la estructura poblacional de la tagua en bosques maduros se caracteriza por una baja densidad de individuos en todos los estadios de crecimiento y la relación entre adultos, subadultos, y juveniles es más equilibrada (15:1, juveniles/adultos), como se observaron en Andil.

Factores ambientales como la luz, la edad del individuo y la estacionalidad influyen en la productividad de hojas e infrutescencias bajo diferentes tipos de manejo. La productividad de hojas es mayor en individuos machos que en hembras. Esto tiene una importante implicación en el manejo ya que se cosechan hojas de las palmas macho (cade) para la elaboración de techados. Las hembras invierten más energía en la producción de grandes infrutescencias, pero producen menos hojas y en promedio de menor tamaño (Montúfar et al., 2013).

Una tagua hembra puede producir hasta 16 infrutescencias anualmente y una infrutescencia puede llegar a pesar 8-15 kilogramos. En el caso de Andil el $50 \%$ de los individuos inventariados presentaban infrutescencias femeninas, el fruto no es manejado por lo que cae y sirve de alimentos a animales del sector.

La muestra representativa (30 palmas) de Phytelephas aequatorialis estudiada en los predios de la granja experimental Andil poseen una estructura poblacional de palmas de tagua con alta desidad de con menos de $1 \mathrm{~m}$ sin tronco visible frente a adultos con tronco visible y con órganos reproductivos, hasta 30:1, presentando ausencia casi total de subadultos, es decir con troncos, pero sin órganos reproductivos. En correspondencia a estos resultados Parrales y Sánchez (2015) efectuaron estudios en el que pudieron conocer el número de jardines botánicos con programas especializados en conservación de palmas es pequeño, lo cual limita el rol que adquiere la conservación ex situ para este grupo y que Ecuador se ubica como uno de los países más diversos

74 UNESUM-Ciencias. Publicación cuatrimestral. Vol. 3, Año 2019, No. 2 (Mayo - Agosto) 
UNESUM-Ciencias: Revista Científica Multidisciplinaria

MICROLOCALIZACIÓN DE Phytelephas aequatorialis Spruce EN LOS PREDIOS DE LA GRANJA EXPERIMENTAL ANDÍL

en palmas a nivel mundial, contabilizándose 134 especies de palmas nativas, de las que al menos 103 tienen algún uso como alimento, medicina o materia prima para construcción y herramientas o especies de palmas que proporcionan productos comerciales.

\section{Resultados de la encuesta dirigida a moradores de la comunidad de Andil, sector de la Granja Experimental, UNESUM}

Los resultados de la encuesta 1relacionados con la frecuencia de las visitas al bosque, el conocimiento, los usos, el aprovechamiento, las partes de la planta que utiliza, así como la creación de un centro de acopio como contribución al desarrollo de su comunidad y la visión de estas personas acerca de formar parte de una asociación, en tanto que cultivan la especie en sus predios, se presentan a continuación.

\section{1.- ¿Visita usted el bosque?}

No se debe hablar de comunidad rural de Ecuador en la que sus pobladores no visiten el bosque con diversos fines dentro de ellos fundamentalmente la recolección de plantas para domesticar o para ser usadas por partes o completamente. De las 10 personas encuestadas, siete visitan el bosque para un 70,0 \%, estos resultados demuestran la dependencia de los pobladores de Andil de los beneficios en bienes y servicios del bosque que les rodea, así como de otras áreas que frecuentan.

\section{2.- ¿Conoce usted la planta de tagua?}

A la interrogante relacionada con el conocimiento de la planta de tagua, el 100\% respondió conocerla, en tanto que solo siete aprovechan esta planta o alguna parte de ella.

\section{3.- ¿Aprovecha usted esta planta o parte de ella? ¿Qué parte de la planta utiliza?}

Los resultados de esta interrogante demuestran que las colectas de plantas o partes de estas en Andil no es factible desde el punto de vista económico debido a que un $40 \%$ de los encuestados no cosechan la planta o parte de ella, lo que redunda en que se pierde más del $90 \%$ del producto. En este aspecto, autores como Jimenez et al., (2017), plantearon que el uso de hojas, frutos o partes del tallo es más sostenible que el de las raíces (si se dañan) o la planta completa. La FAO, ha planteado que en todo el mundo existe dependencia de los Productos Forestales No Madereros (PFNM), para su subsistencia y para la obtención de ingresos. Alrededor del 80 por ciento de la población del planeta, en particular la de los países en desarrollo, utiliza los PFNM para satisfacer necesidades nutricionales y de salud.

A nivel local, los PFNM también se utilizan como materia prima para la elaboración industrial a gran escala. Varios PFNM son objeto de comercio internacional. Actualmente, hay al menos 150 PFNM que tienen importancia en el comercio internacional, entre ellos la miel, la goma arábiga, el bambú, el corcho, las nueces y hongos, las resinas, los aceites esenciales, y partes de plantas y animales para obtener productos farmacéuticos. Recientemente los PFNM han suscitado un interés considerable por su importancia cada vez más reconocida y la consecución de objetivos ambientales como la conservación de la diversidad biológica (FAO, 2014 y 2017). Los resultados aquí obtenidos no se corresponden con el aprovechamiento que se le da a esta especie en otras partes de Ecuador.

Para la UICN (2017), el valor económico potencial de la especie Phytelephas aequatorialis hace que la protección de las últimas poblaciones silvestres sea una alta prioridad, a fin de preservar la variabilidad genética. La principal amenaza es la sobreexplotación de la fruta; la infrutescencia se 
John Ricardo Pin Cedeño, Alfredo Jimenez González, Marcos Pedro Ramos Rodríguez...

cosecha entera cuando está madura, dejando el árbol estéril. Clasificado como raro en 1997 por la Unión Internacional para la Conservación de la Naturaleza (UICN). Casi califica para el listado como Vulnerable bajo el criterio A.

\section{4.- ¿Con que frecuencia se dirige al bosque con la finalidad de aprovechar la tagua?}

De acuerdo con los resultados de esta interrogante, la dependencia de los pobladores de Andil de los beneficios en bienes y servicios del bosque que les rodea, corrobora los criterios de Wong et $a l .$, (2001), estos autores plantearon que el rendimiento sostenible es la utilización de los recursos vivos con niveles y formas de aprovechamiento que les permite proporcionar productos y servicios por tiempo indefinido. Significa además la extracción del interés, en lugar del capital, de una base de recursos.

\section{5.- ¿Qué usos le da a la parte de la planta que utiliza?}

En el caso de la pregunta relacionada con los usos que le dan los moradores de Andil a la planta de Phytelephas aequatorialis, la mayoría se inclinó por la recolección de los frutos, y en menor porcentaje utilizan las hojas para el techado de sus viviendas y otras instalaciones como chozas.

Los frutos y las hojas de Phytelephas aequatorialis constituyen las partes fundamentales que recolectan los pobladores de Andil, utilizados para alimento y para construcción, como la mococha y el cade, respectivamente.

A la pregunta sobre el conocimiento de los beneficios de la planta, el $60 \%$ de los encuestados respondió afirmativamente. El hecho de que el $40 \%$ desconozca sobre la planta de tagua y sus beneficios, puede estar influyendo en que no la aprovechen como un recurso de su entorno.

Los resultados de la pregunta vinculada con las actividades de aprovechamiento de la planta o partes de esta, evidenciaron que la mayor cantidad de encuestados aprovecha los frutos para venderlos, en tanto que el resto utilizan las hojas. Llama la atención que casi la mitad de las personas no usan la planta, aspecto que puede estar indicando la necesidad de proyectar acciones para el aprovechamiento sostenible de esta especie en la zona.

\section{6.- ¿Además de cosechar la tagua, se dedica usted a sembrar la especie?}

Esta es una de las interrogantes que demostró la falta de incentivos para el fomento, cultivo, aprovechamiento y comercialización de productos derivados de la especie Phytelephas aequatorialis, lo constituye el hecho de que ninguno de los encuestados se dedique a cultivar esta especie, lo que justifica el planteamiento de promover estrategias de conservación y aprovechamiento de los productos forestales no maderables, con énfasis en la tagua, en la zona de Andil.

\section{7.- ¿Cree usted que la creación de un centro de acopio contribuye positivamente al desarrollo?}

Todas las personas encuestadas, incluso las personas que desconocían del manejo de la tagua coincidieron en que la creación de un centro de acopio en su comunidad llamaría la atención de los agricultores del sector y aseguraron le tomarían más importancia, ya que abaratarían los costos de transportación de los productos y por ende trabajarían más en la explotación responsable de la palma.

\section{8.- ¿Piensa usted que sería beneficioso formar parte de una asociación? ¿Por qué?}


UNESUM-Ciencias: Revista Científica Multidisciplinaria

MICROLOCALIZACIÓN DE Phytelephas aequatorialis Spruce EN LOS PREDIOS DE LA GRANJA EXPERIMENTAL ANDÍL

Todas las personas encuestadas concluyeron que formar parte de una asociación les permitiría tener acceso a capacitaciones y tecnificación sobre el manejo de los recursos obtenidos de la tagua, y que sería un aporte importante ya que verían en la asociación un medio del cual se puedan apoyar desde la recolección hasta conseguir un precio justo en el momento de la venta de la materia prima.

\section{9.- ¿Actualmente pertenece a alguna asociaron? ¿Cuál es?}

Según los datos obtenidos de esta pregunta es claro que los comuneros de este sector no pertenecen a ningún tipo de asociación que les permita orientarse en el desarrollo de sus habilidades para la cultivación de la tagua.

\section{Resultados de la encuesta dirigida a los centros de acopio de productos de Phytelephas aequatorialis}

\section{1.- ¿Cree usted que la materia prima que llega a sus centros de acopio es de buena calidad?}

La comercialización de productos forestales no maderables (PFNM) ha sido ampliamente promocionada como un aporte al desarrollo rural en las áreas forestales tropicales. A fin de asegurar que los PFNM aporten todo su potencial para el desarrollo sostenible, es preciso entender las razones que llevan al éxito y al fracaso de la comercialización de PFNM, y las condiciones bajo las cuales ésta puede contribuir en forma positiva al mejoramiento de la calidad de vida de los pobres (Marshall et al., 2006).

Las personas encuestadas en establecimientos de Manabí, se mostraron interesados y preocupados a esta interrogante, la mayoría estuvo de acuerdo en decir que la materia prima que llega es excelente, otros que es buena, en general perciben que la calidad de las materias que llegan es buena. Algunos autores han planteado que la cadena de valor de PFNM es altamente dinámica. Así los productores, los procesadores y los comerciantes muestran un alto grado de resiliencia al estrés externo y una gran habilidad de adaptación a los cambios de contexto.

La respuesta a la interrogante relacionada con el acceso a los servicios básicos, electricidad y agua de los talleres de procesamiento de la tagua, demostró que en el $100 \%$ de esos centros existen estos servicios, lo que dinamizaría en este caso los servicios al comprar y vender el producto, pudiendo implementar equipos de metrología y control de la calidad como por ejemplo balanzas grameras que funcionen con electricidad.

\section{3.- ¿Considera usted que deberían recibir capacitaciones que les indique como aprovechar de mejor manera la tagua?}

En relación con el tema de las capacitaciones, de manera que transmitan el cómo aprovechar mejor la tagua, la mayoría de los encuestados afirma estar muy de acuerdo en recibir estos servicios. De acuerdo con varios autores, el mejoramiento funcional cambia la combinación de las actividades realizadas en una cadena de valor o empresa individual. Por ejemplo, asociaciones cooperativas han asumido nuevas funciones a través de la provisión de crédito, la capacitación y el mercadeo. Los resultados obtenidos en esta investigación indican que los dueños de estos negocios sienten deseos de superación y ganas de aprender sobre el manejo de la materia prima, también sobre cómo manejarla y cómo sacar un mejor provecho de ella.

\section{4.- ¿Cuál es su percepción sobre la frecuencia de llegada de vendedores de tagua a su centro de acopio?}


John Ricardo Pin Cedeño, Alfredo Jimenez González, Marcos Pedro Ramos Rodríguez...

La frecuencia con que llegan algunos vendedores de la tagua a estos centros puede estar influenciando en la calidad del producto final, así también la repercusión que esto tendría sobre el precio final de la mercadería.

Según estos resultados casi la tercera parte de las personas encuestadas estuvieron de acuerdo en que la llegada de vendedores de tagua a sus centros de acopio es frecuente, el resto manifestaron que es indiferente, es decir es muy poca la llegada de vendedores de tagua a diferencia de otros productos como el maíz y el café.

\section{5.- ¿Se siente satisfecho con la remuneración que recibe por su trabajo?}

Al respecto de las respuestas a esta interrogante, Marshall, Schreckenberg y Newton (2006), plantearon que una cadena de valor de PFNM exitosa puede presentarse de diferentes maneras, que van de las cadenas cortas locales con conexiones directas entre productores y consumidores, a las cadenas largas internacionales con varios intermediarios. En consecuencia, estos autores concluyeron que la fijación transparente de precios y los términos de negociación son factores clave para asegurar la satisfacción de los productores pobres. Los porcentajes de satisfacción oscilan sobre los 70 puntos porcentuales de personas encuestadas satisfechos con la remuneración que reciben porque les alcanza para subsistir, en tanto que sienten que podrían mejorar, el resto de los encuestados asegura sentirse indiferente el ingreso que reciben por ventas de la tagua ya que venden muy poco y se enfocan más en otros productos.

\section{6.- ¿Cuál es su percepción sobre si se incentiva en el agricultor la cultura de cultivar la tagua?}

Acerca de la percepción que tienen estas personas de los centros de acopio, sobre si el agricultor se siente incentivado a trabajar la tagua, ejemplo plantar, cultivar, extender esta especie se concluye que no conocen o no han hecho conexiones con los habitantes de esas comunidades, ya que el 70 $\%$ de los encuestados no motiva al agricultor a seguir produciendo, el resto entre un 20 y un 10 puntos porcentuales plantearon que si lo incentiva al productor en las compras de sus productos, esta puede ser una de las causas por las que también haya poco interés de parte de los productores de la materia prima en cosechar la tagua. Estos resultados corroboran lo descrito por Cordero, Moreno y Kosmus (2008), quienes aseguraron que muchos bienes y servicios ambientales carecen de un precio y cómo la economía ambiental ha desarrollado una serie de metodologías para dar un valor económico a los bienes, servicios e impactos ambientales. Lo que permite contar con un indicador de su importancia para la sociedad.

\section{7.- ¿Considera que su trabajo es valorado?}

Otra de las temáticas recurrentes en esta encuesta es la pregunta relacionada con lo que considera un trabajador de los centros de acopio sobre si su trabajo es valorado, a los que el $70 \%$ respondió afirmativamente, no obstante, agregan que no como ellos quisieran, mientras que el resto de los encuestados dijeron sentirse indiferentes y afirman que sienten que su trabajo es poco valorado.

\section{8.- ¿Estaría de acuerdo en crear una asociación que apoye al trabajo conjunto de la tagua?}

En relación con la pregunta de si estaría de acuerdo en crear una asociación que apoye al trabajo conjunto de la tagua, el 100\% de los encuestados respondieron estar de acuerdo o muy de acuerdo. Alegaron que la creación de una asociación ayudaría al trabajo conjunto a los agricultores, otros manifestaron que sería óptimo pues se mejorarían las relaciones entre los vendedores y compradores de la tagua y el trabajo conjunto sería mucho más eficiente. 
UNESUM-Ciencias: Revista Científica Multidisciplinaria

MICROLOCALIZACIÓN DE Phytelephas aequatorialis Spruce EN LOS PREDIOS DE LA GRANJA EXPERIMENTAL ANDÍL

9.- ¿Considera que su comprador de materia prima se siente satisfecho con la calidad de la tagua?

Sobre lo que piensan los encuestados de los centros de acopio, sobre la satisfacción de los compradores o del comprador de la materia prima en relación con la calidad de la tagua que le venden, estos respondieron percibir que su producto es bueno, ya que el $100 \%$ aseguró sentirse satisfecho o muy satisfecho con la salida del producto, demostrado con la demanda de tagua que tienen de sus clientes.

\section{0.- ¿Le gustaría tener apoyo del gobierno para mejorar su entorno laboral?}

Sobre el tema de apoyo gubernamental el $100 \%$ de los encuestados respondió afirmativamente a la necesidad de tener apoyo del gobierno para mejorar su entorno laboral. Para estas personas seria de suma importancia contar con apoyo gubernamental no solo para mejorar el entorno laboral, sino para capacitarlos y de esta manera crear una cadena de educación sobre el aprovechamiento de la tagua y transmitir esas enseñanzas y seguridad laboral a los agricultores y así poder potenciar el aprovechamiento de la tagua en la zona. Al respecto Cordero, Moreno y Kosmus (2008), concuerdan con que la preocupación por la conservación de los recursos naturales ha ganado importancia en los últimos años.

Resultados de la encuesta dirigida a procesadores y comerciantes de Phytelephas aequatorialis, con el objetivo de indagar sobre la manufactura y comercialización de PFNM derivados de esta planta en sectores de Manabí

\section{1.- ¿Es la comercialización de artesanías hechas en tagua su principal fuente de ingresos?}

La primera pregunta relacionada con la comercialización de artesanías hechas en tagua, indagó si es su principal fuente de ingresos. A lo que el $100 \%$ de los encuestados respondió afirmativo, constituyendo su principal fuente de ingresos económico devenida en la elaboración de productos hechos en tagua y posterior venta de estos, con lo que han podido sostener sus hogares y darles alimentación y estudio a sus hijos.

2.- ¿Cómo comerciante le gustaría exportar productos derivados de la tagua al mercado internacional?

E1 $100 \%$ de los encuestados respondió que les gustaría ser exportadores directos al mercado internacional de sus productos, ya que normalmente el intermediario es quien se lleva la gran parte de los ingresos y ellos se sienten afectados en esto. Hernández \& Celi (2015), plantearon que las condiciones para exportar productos artesanales son las más acogidas en el mundo entero porque son considerados más que un intercambio comercial como un intercambio cultural entre países, debido en que cada pieza se plasma la creatividad de los artesanos que son capaces de transformar cualquier materia prima en bella bisutería.

\section{3.- ¿Piensa usted que las artesanías que comercializa pueden calificarse de buena calidad?}

De acuerdo con los resultados de esta pregunta el $100 \%$ de los encuestados manifestaron que las artesanías que comercializan son de excelente calidad, comprobado a través de la oferta y demanda de las artesanías. Las opiniones están distribuidas en que solo a veces utilizan estrategias para promocionar sus productos, otros casi siempre lo hacen, un menor porcentaje aduce siempre usar estrategias de marketing para vender sus productos, el resto asegura que no usa nunca estrategias de mercadeo pero que así le va bien en sus ventas. 
John Ricardo Pin Cedeño, Alfredo Jimenez González, Marcos Pedro Ramos Rodríguez...

\section{4.- ¿Cree usted que la venta de productos de tagua contribuye al desarrollo de su familia?}

A la interrogante sobre las ventas de la tagua en el sustento familiar los encuestados respondieron, la mayoría, que siempre ayuda al desarrollo de las familias. Para ellos la venta de artesanías hechas en tagua es su principal fuente de ingresos económicos y por ende contribuyen sin lugar a dudas al desarrollo de su familia, mientras que el resto (30\%), aseguró que reciben buena remuneración de la venta de sus artesanías.

\section{5.- ¿Sabe usted como utilizar el internet para promocionar su mercadería?}

Los resultados de esta pregunta han diferido, entre casi siempre y muy pocas veces, la mayoría de los encuestados manifestaron que muy pocas veces usan el internet para poder promocionar sus artesanías, mientras tanto un 30\% asegura que a veces accede a la web para de una u otra manera promocionar sus mercaderías, los demás, aseguran que casi siempre está pendiente de redes sociales o sitios web donde pueda hacer conocer los productos que ofrece.

\section{6.- ¿Le gustaría a usted que lo capacitaran en técnicas de marketing para promocionar sus productos?}

De acuerdo con los resultados de esta pregunta la totalidad de los encuestados manifiesta que siempre es bueno contar con cursos de capacitación que les permita conocer las técnicas actuales de marketing y así poder entender la situación del mercado actual y como ellos puedan desarrollarse de mejor manera para poder ofrecer sus artesanías al mundo entero. Lo cual corrobora lo evidenciado en la anterior pregunta sobre el acceso a internet, por la estrecha relación que tienen el acceso a internet y una buena estrategia de marketing.

\section{7.- ¿Piensa usted que las artesanías que vende son correctamente valoradas?}

En relación con la percepción que tienen los comerciantes sobre la valoración de sus artesanías, el $50 \%$ de los comerciantes de Manabí respondieron estar satisfechos, siempre o casi siempre, en tanto que el resto solo a veces.

\section{8.- ¿Utilizan equipos de seguridad industrial para proteger la salud de sus obreros?}

Aquí los resultados arrojan un 50\% de los encuestados respondieron: solo a veces utilizan equipos de seguridad industrial el justificativo es que ya están acostumbrados a su trabajo y normalmente no ocurren accidentes, un $40 \%$ dijo que utilizan equipos de seguridad industrial para salvaguardar su salud al momento de trabajar la tagua y un $10 \%$ respondió que muy pocas veces usan protección al trabajar.

Por otra parte en la Conferencia General de la Organización Internacional del Trabajo convocada en Ginebra por el Consejo de Administración de la Oficina Internacional del Trabajo; citado como el Convenio sobre seguridad y salud de los trabajadores, 1981, estableció en su artículo 3 los términos a tener en cuenta por los empleados y empleadores públicos, y del cual Ecuador es signatario.

\section{9.- ¿Le gustaría formar parte de alguna asociación que le ayudara a exportar sus artesanías?}

Los resultados de esta pregunta ofrecen que para el $70 \%$ de los encuestados siempre sería beneficioso contar con apoyo de alguna organización que les ayude a exportar sus productos y así obtener una mejor rentabilidad, el 30\% restante dijo estar de acuerdo, pero con ciertas dudas de cómo se manejarían las ventas y los precios, recalcaron todos que son miembros de una asociación llamada TROPITAGUA a la cual pertenecen actualmente 23 personas donde cuentan con 

EXPERIMENTAL ANDÍL

herramientas como impresoras láser y tornos de moldeo electrónico 3D que, además de modelar tagua también pueden utilizarlo para madera, supieron manifestar que no lo usan por falta de capacitación.

En los estudios de comercialización realizados por Vélez \& Doval, (2017), Ecuador y sobre todo la región de Manabí en la actualidad se caracteriza por la producción artesanal de la tagua. Esta actividad económica abarca diversas parroquias y comunidades en la que intervienen numerosos y diferentes actores sociales. En Manabí laboran alrededor de 35 mil personas en torno a la producción de la tagua, ya que tiene excelente cotización y demanda a nivel nacional e internaconal, lo que confirma los resultados del presente estudio.

Sin embargo indagaciones realizada por Pánchez et al., (2017) demostraron que desde que se realiza el acopio hasta su comercialización existen algunas falencias, especialmente de apoyo Estatal en el proceso productivo de los diferentes derivados de tagua (Phytelephas aequatorialis), en relación a lo anteriormente descrito, las respuestas que dieron los comuneros de Andil, que no explotan la población de tagua del lugar, porque trasportarla desde el lugar de cosecha genera más costos que la venta del producto, así mismo sucede con el proceso de comercialización, existiendo gran demanda del producto, pero los artesanos mantienen varios impedimentos de capacitación, burocráticos y de logística a la hora de satisfacer la demanda del mercado internacional.

Pese a los inconvenientes detectados se estima que Ecuador mantiene un gran potencial como exportador de productos de tagua, ya que es considerado un país privilegiado porque la Phytelephas aequatorialis (tagua) crece de manera silvestre especialmente en la región Costa del país, existiendo la oportunidad de convertirse en uno de los principales exportadores de América Latina y de la Unión Europea, beneficiando directamente al sector rural y artesano del país.

\section{CONCLUSIONES}

Las prácticas tradicionales de uso y aprovechamiento de los bosques, han modificado las poblaciones de Phytelephas aequatorialis, en los predios de Andil, no obstante, los especímenes inventariados cumplen las características morfológicas típicas de la especie.

El aprovechamiento y el procesamiento de productos forestales no madereros derivados de Phytelephas aequatorialis no es sustentable, en los centros encuestados en Manabí, en tanto que las artesanías de la tagua están posicionadas en el mercado nacional e internacional.

\section{REFERÉNCIAS BIBLIOGRÁFICAS}

Cordero, D.; Moreno, A. y Kosmus, M. (2008). Manual para el desarrollo de mecanismos de pago/compensación por servicios ambientales. Equipo Regional de Competencia y Programa GESOREN, GTZ-Ecuador.

Eke, J.; Gretzinger, S.; Camacho, O.; Sabogal, C. y Arce, R. (2016). Desarrollo Forestal Empresarial por Comunidades. Guía práctica para promotores forestales comunitarios en los trópicos Américanos. Organización de las Naciones Unidas para la Alimentación y la Agricultura (FAO) y Centro Agronómico Tropical de Investigación y Enseñanza (CATIE). p. 25

Grijalva, J. Checa, R. Ramos, P. Barrera y R. Limongi. (2012). Situación de los Recursos Genéticos Forestales Informe País Ecuador. Preparado por el Programa Nacional de Forestería del INIAP con aval del INIAP/FAO/MAE/MAGAP/MMRREE. Recuperado el 14 de 02 de 2017, de http://www.iniap.gob.ec/nsite/images/stories/descargas/informe_pas_rgf_ecuador_final_.pdf 
John Ricardo Pin Cedeño, Alfredo Jimenez González, Marcos Pedro Ramos Rodríguez...

Hernández, K. S. y Celi, K. P. (2015). Plan de exportación de bisuterías a base de tagua producidas en el cantón Rocafuerte para el mercado de Nueva York de los estados unidos de América. Tesis en opción al título de ingeniero Comercial con Mención en Comercio Exterior. Universidad politécnica Salesiana del Ecuador, 128 p.

Isasi-Catalá, E. (2011). Los Conceptos de Especies Indicadoras, Paraguas, Banderas y Claves: Su Uso y Abuso en Ecología de la Conservación. Interciencia; Jan , 36 (1): 31-38.

INABIO. (2017). Agenda nacional de investigación sobre la biodiversidad. MAE, SENESCYT e INABIO. Quito. 20 pp. ISBN: 978-9942-9988-9-7

Jimenez, A. (2012). Contribución a la ecología del bosque semideciduo mesófilo en el sector oeste de la Reserva de la Biosfera "Sierra del Rosario", orientada a su conservación. 111 p. Tesis en opción al grado científico de Doctor en Ciencias Forestales. Universidad Hermanos Saiz Montes de Oca.

Jiménez, A., Pincay, F.A., Ramos, M.P., Mero, O.F., Cabrera, C.A. (2017). Utilización de productos forestales no madereros por pobladores que conviven en el bosque seco tropical. Revista Cubana de Ciencias Forestales; sept. - dic. 2017 Vol. 5(3):270-286. ISSN: 1996-2452 RNPS: 2148. Recuperado de: http://cfores.upr.edu.cu/index.php/cfores/article/view/264/html

Marshall, E., Schreckenberg, K. y Newton, A.C. (Eds). (2006). Comercialización de ProductosForestales No Maderables: Factores que Influyen en el Éxito. Conclusiones del Estudio de México y Bolivia e Implicancias Políticas para los Tomadores de Decisión. Centro Mundial de Vigilancia de la Conservación del PNUMA, Cambridge, Reino Unido.

Ministerio del Ambiente (MAE). (2012). Sistema de clasificación de los ecosistemas del Ecuador continental. Subsecretaría de Patrimonio Natural. Quito, Ecuador. Recuperado el 02 de 03 de 2017, de http://www.ambiente.gob.ec/wp-content/uploads/downloads/2012/09/LEYENDA-

ECOSISTEMAS_ECUADOR_2.pdf

Morales, P. (2012). Estadística aplicada a las Ciencias Sociales tamaño necesario de la muestra: ¿Cuántos sujetos necesitamos? .Universidad Pontíficia Comillas. Madrid. Facultad de Humanidades (Última revisión, 13 de Diciembre, 2012). Madrid, España.

Montúfar, R., Brokamp, G., Jácome, J. (2013). Tagua. Phytelephas aequatorialis. En: Palmas Ecuatorianas: biología y uso sostenible (R. Valencia, R. Montúfar, H. Navarrete, y H. Balslev, eds) pp. 187-201. Publicaciones del Herbario QCA, Pontificia Universidad Católica del Ecuador. ISBN: 978-9942-13-263-5.

Organización de las Naciones Unidas para la Alimentación y la Agricultura. (2014). Que son los PFNM? FAO recuperado de http://www.fao.org/forestry/nwfp/6388/es/.

Organización de las Naciones Unidas para la Agricultura y la Alimentación (FAO). (2017). Agenda público privado para el desarrollo sostenible de los productos forestales no madereros en Chile. Chile: Consejo de politica forestal, $\quad$ recuperado

https://www.infor.cl/images/pdf/Libro_Agenda_PFNM_Consejo_de_Politica_Forestal.pdf.

Pánchez, J. P., Vargas, J. G., Rodríguez, K. V., \& Polo, I. C. (2017). Estudio para la creación del centro de procesamiento y taller artesanal para la elaboración de productos derivados del marfil vegetal (tagua), en la comuna las Núñez provincia de Santa Elena. Revista Científica y Tecnológica UPSE, 4(1), 7-15.

Parrales, F. S., \& Bravo-Sánchez, S. (2015). Evaluación de la colección de palmas del jardin botanico Padre Julio Marrero (Ecuador) y su propuesta de conservación de las especies nativas. Rev. Inclusiones, recuperado de http://www.revistainclusiones.com/gallery/13\%20oficial\%20articulo\%202015\%20oct\%20dic\%20\%20\%20 2015\%20rev\%20inc.pdf.

Santos, G. A.; Batugal, P . A.; Othman, A.; Baudouin, L. y Labouisse, J. P. (Ed.) (s/f). Manual sobre Técnicas Estandarizadas para la Investigación del Mejoramiento del Cocotero. IPGRI (International Plant Genetic Resources Institute), COGENT. 94 p.

Torres, M., Paz, K., y Salazar, F. (2006). Tamaño de una muestra para una investigación de mercado. Universidad Rafael Landívar: Boletín electrónico [en línea]. Guatemala. doi:http://www. tec. url. edu. gt/boletin/URL_02_BAS02.pdf.

82 UNESUM-Ciencias. Publicación cuatrimestral. Vol. 3, Año 2019, No. 2 (Mayo - Agosto) 
UNESUM-Ciencias: Revista Científica Multidisciplinaria

ISSN 2602-8166

MICROLOCALIZACIÓN DE Phytelephas aequatorialis Spruce EN LOS PREDIOS DE LA GRANJA EXPERIMENTAL ANDÍL

Unión Internacional para la Conservación de la Naturaleza y los Recursos Naturales (UICN), (2017). The IUCN Red List of Threatened Species (tm) 2017-3 . Recuperado de: http://www.iucnredlist.org/details/43981/0

Valencia, R.; Montúfar, R.; Navarrete, H. y Balslev, H. (Ed.) (2013). Palmas Ecuatorianas: biología y uso sostenible, . ISBN: 978-9942-13-263-5. Recuperado de: https://www.researchgate.net/publication/259823093_Capitulo_13_Tagua_Phytelephas_aequatorialis.

Wong, J., Thornber, K., y Baker, N. (2001). Evaluación de los Recursos de Productos Forestales no Maderos. Experiencias y Principios Biométricos, ( $\left.\mathrm{N}^{\mathrm{o}} 13\right)$. Organización de las Naciones Unidas para la Agricultura y la Alimentación (FAO). Roma. Roma, Italia. Recuperado el 27 de 02 de 2017, de http://www.fao.org/3/ay1457s.pdf.

Vélez, N. J., \& Doval, Y. R. (2017). Análisis prospectivo del proceso de reconversión en la comunidad . ReHuSo: Revista de Ciencias Humanísticas y Sociales, recuperado de https://revistas.utm.edu.ec/index.php/Rehuso/article/view/1252/1036. 
John Ricardo Pin Cedeño, Alfredo Jimenez González, Marcos Pedro Ramos Rodríguez...

84 UNESUM-Ciencias. Publicación cuatrimestral. Vol. 3, Año 2019, No. 2 (Mayo - Agosto) 